\title{
ON QUASI-DECOMPOSITIONS OF TORSION FREE ABELIAN GROUPS
}

\author{
J. D. REID ${ }^{1}$
}

1. Introduction. In this paper we establish a correspondence between quasi-decompositions of certain torsion free Abelian groups and decompositions of their quasi-endomorphism rings into left ideals. (For definitions of these terms, which are taken from $[1 ; 4$ and 7], see §2.) The groups we discuss are those whose quasi-endomorphism rings have minimum condition on left ideals and thus include the groups of finite rank. The theorem we obtain is applied in $\$ 4$ to prove an analog of the Krull-Schmidt Theorem which generalizes a result of Jónsson [4] and to prove an analog of Fitting's Lemma.

The paper had its origin in a suggestion of Professor R. S. Pierce that a proof of Jónsson's Theorem might be based on the discussion of the Krull-Schmidt Theorem given in Jacobson [5] and represents a revision of a part of the author's Ph.D. thesis at the University of Washington. The author would like to thank Professor Pierce for suggesting the problem and for his help and encouragement in the preparation of the paper. He would also like to thank the referee whose suggestions resulted in a substantial simplification of the proofs and a very much improved presentation.

All groups considered are torsion free Abelian groups and we assume that they are subgroups of a fixed rational vector space $V$. We denote the ring of linear transformations of $V$ by $\mathscr{L}(V)$. A subgroup $G$ of $V$ will be said to be full in $V$ if $V / G$ is a torsion group; equivalently, if $V$ is the minimal divisible group containing $G$.

\section{Preliminaries.}

Definition 2.1. Let $A$ and $B$ be any two subgroups of $V$. We say that

(i) $A$ is quasi-contained in $B(A \subseteq B)$ if $n A \subseteq B$ for some nonzero integer $n$.

(ii) $A$ and $B$ are quasi-equal $(A \doteq B)$ if $A \subseteq B$ and $B \subseteq A$.

(iii) $A$ and $B$ are quasi-isomorphic $(A \stackrel{\cong}{\cong})$ if there are isomorphic subgroups $A^{\prime}$ and $B^{\prime}$ of $V$ such that $A \doteq A^{\prime}$ and $B \doteq B^{\prime}$.

The relations defined on the set of subgroups of $V$ by (ii) and (iii)

\footnotetext{
Presented to the Society, January 25, 1961 ; received by the editors October 28, 1960 and, in revised form, June 5, 1961.

1 This work was supported by the National Science Foundation Research Grant NSF-G11098.
} 
above are equivalence relations and the relation defined in (i) is clearly reflexive and transitive. Furthermore, the subgroups $A$ and $B$ of $V$ are quasi-isomorphic if and only if $B$ is isomorphic to a subgroup of $A$ which is quasi-equal to $A$. These facts are proved in [1].

Definition 2.2. We will say that the subgroup $A$ of $V$ is quasidecomposable if there exist nonzero independent subgroups $B$ and $C$ of $V$ such that $A \doteq B \oplus C$. If $A$ is not quasi-decomposable, $A$ will be said to be strongly indecomposable.

Definition 2.3. Let $G$ be a full subgroup of $V$. Put

$$
E(G)=\{\lambda \in \mathscr{L}(V) \mid \lambda(G) \subseteq G\} .
$$

The elements of $E(G)$ will be called quasi-endomorphisms of $G$. If $\lambda$ is a unit of $\mathscr{L}(V)$ and both $\lambda$ and $\lambda^{-1}$ belong to $E(G)$ then $\lambda$ will be called a quasi-automorphism of $G$.

It follows from these definitions that $E(G)$ is a subring of $\mathscr{L}(V)$ and that an element $\lambda \in \mathfrak{L}(V)$ is a quasi-automorphism of $G$ if and only if $\lambda(G) \doteq G$. It is also easy to prove.

LEMma 2.4. Let $G$ and $G^{\prime}$ be full subgroups of $V$. If $G \doteq G^{\prime}\left(G \cong G^{\prime}\right)$ then $E(G)=E\left(G^{\prime}\right)\left(E(G) \cong E\left(G^{\prime}\right)\right)$.

In Definition 2.3, we have defined $E(G)$ relative to a minimal divisible group $V$ containing $G$. It is clear that $E(G)$ is uniquely determined up to isomorphism by $G$. In the lemma below, by a proper idempotent we mean an idempotent $e \neq 0,1$. The lemma follows directly from the definitions.

LEMMA 2.5. If $e$ is an idempotent element of $E(G)$, then $G \doteq H_{1} \oplus H_{2}$ where $H_{1}=e(V) \cap G \doteq e(G)$ and $H_{2}=(1-e)(V) \cap G \doteq(1-e)(G)$. Conversely, if $G \doteq H_{1} \oplus H_{2}$, there is a unique idempotent $e \in E(G)$ such that $H_{1} \doteq e(G), H_{2} \doteq(1-e)(G)$. Thus, $G$ is strongly indecomposable if and only if $E(G)$ contains no proper idempotents.

3. The Main Theorem. Where proofs have been omitted below, the results are either well known [6, pp. 50-51] or follow easily from well-known facts.

LEMMA 3.1. Let e and f be nonzero idempotents in $E(G)$. Let $W=e(V)$, $U=f(V)$. Let $E(e(G), f(G))=\{\phi \in \operatorname{Hom}(W, \quad U) \mid \phi(e(G)) \subseteq f(G)\}$. Then the restriction mapping $\lambda \rightarrow \lambda \mid W$ sends $f E(G) e$ one-to-one onto $E(e(G), f(G))$.

Proof. Put $W^{\prime}=(1-e)(V)$. It is easy to see that the elements of $f E(G) e$ are just those $\lambda \in \mathscr{L}(V)$ such that $\lambda \mid W \in E(e(G), f(G))$ and $\lambda \mid W^{\prime}=0$. This proves the lemma. 
THEOREM 3.2. Let e be a nonzero idempotent of $E(G)$. Then the rings $E(e(G))$ and $e E(G) e$ are isomorphic to each other and anti-isomorphic to $\operatorname{Hom}_{E(G)}(E(G) e, E(G) e)$.

Proof. That $E(e(G)) \cong e E(G) e$ follows from Lemma 3.1 and the observation that $E(e(G)) \cong E(e(G), e(G))$. The second statement is well-known [6, p. 51].

Corollary 3.3. Let $e$ be a nonzero idempotent of $E(G)$. Then the following are equivalent conditions: (i) $e(G)$ is strongly indecomposable; (ii) $e E(G) e$ has no proper idempotents; (iii) $E(G) e$ is indecomposable as an $E(G)$ module.

Proof. The equivalence of (i) and (ii) follows from Theorem 3.2 and Lemma 2.5. That (ii) and (iii) are equivalent is well known.

THEOREM 3.4. Let $e$ and $f$ be nonzero idempotents of $E(G)$. Then

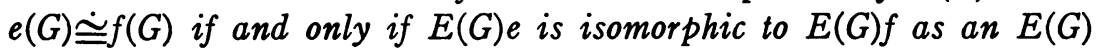
module.

Proof. It is well known [6, p. 51] that $E(G) e \cong E(G) f$ if and only if $u, v$ exist in $E(G)$ such that $u \in e E(G) f, v \in f E(G) e, e=e u v$ and $f=f v u$. By Lemma 3.1 this is equivalent to the existence of $\phi \in E(f(G), e(G))$ and $\psi \in E(e(G), f(G))$ such that $\phi=\psi^{-1}$, or in other words, to $e(G)$ $\stackrel{\cong}{\cong}(G)$.

THEOREM 3.5. Suppose that $E(G)$ has the minimum condition on left ideals. Then any decomposition $G \doteq \sum_{\lambda \in \Lambda} \oplus H_{\lambda}$ of $G$ into nonzero subgroups has only finitely many summands. The correspondence

$$
G \doteq \sum_{i=1}^{k} \oplus e_{i} G \rightarrow E(G)=\sum_{i=1}^{k} \oplus E(G) e_{i} \quad\left(e_{i} \text { idempotent }\right)
$$

between the decompositions of $G$ and the decompositions of $E(G)$ into a direct sum of left ideals is one-to-one.

Proof. Let $G \doteq \sum_{\lambda \in \Lambda} \oplus H_{\lambda}$. By Lemma 2.4, $E(G)=E\left(\sum_{\lambda \in \Lambda} \oplus H_{\lambda}\right)$ so the projections $e_{\lambda}$ of this decomposition belong to $E(G)$. Since $1 \in E(G)$ and for each $\lambda, H_{\lambda}$ is nonzero, it follows that $L_{\lambda}=E(G) e_{\lambda}$ is a nonzero left ideal of $E(G)$. It is clear that the sum $\sum_{\lambda \in \Delta} L_{\lambda}$ is direct. Minimum condition on $E(G)$ implies that $\Lambda$ is finite. We now have $1=\sum_{\lambda \in \Delta} e_{\lambda}$ since this is true on $\sum_{\lambda \in \Lambda} \oplus H_{\lambda}$ which is full in $V$. Thus, $E(G)=\sum_{\lambda \in \Delta} \oplus L_{\lambda}$.

Now suppose that $E(G)=\sum_{i=1}^{k} \oplus L_{i}$ with each $L_{i}$ a nonzero left ideal of $E(G)$. Then, as is well known, where exist orthogonal idempotents $e_{i}$ such that $L_{i}=E(G) e_{i}(i=1, \cdots, k)$. By an obvious ex- 
tension of Lemma 2.5 we have $G \doteq \sum_{i=1}^{k} \oplus e_{i}(G)$. It is now clear that the correspondence is one-to-one.

4. Applications. In this section we assume that $E(G)$ has the minimum condition on left ideals.

Theorem 4.1. Let $\sum_{i=1} \oplus H_{i} \doteq G \doteq \sum_{j=1}^{t} \oplus K_{i}$ with $H_{i}$ and $K_{j}$ strongly indecomposable $(i=1, \cdots, s ; j=1, \cdots, t)$. Then $s=t$ and for some permutation $\pi$ of $\{1, \cdots, t\}$, we have $H_{i} \doteq K_{x(i)}(i=1, \cdots, t)$.

Proof. Let $E(G)=\sum_{i=1}^{i} \oplus L_{i}$ and $E(G)=\sum_{j=1}^{t} \oplus M_{j}$ be the decompositions of $E(G)$ associated with the given decompositions of $G$ by Theorem 3.5. Then, by Corollary 3.3 , the $L_{i}$ and $M_{j}$ are indecomposable $E(G)$ modules. Since $E(G)$ has minimum condition on left ideals and the additive group of $E(G)$ is torsion free, $E$ also has maximum condition on left ideals (see [2, p. 285]). By the KrullSchmidt Theorem for operator groups, we conclude that $s=t$ and for each $i$ there is an $E(G)$-isomorphism mapping $L_{i}$ onto $M_{\pi(i)}$ where $\pi$ is some permutation of $\{1, \cdots, t\}$. By Theorem 3.4 this implies that $H_{i} \doteq K_{x(i)}(i=1, \cdots, t)$ and the proof is complete.

If the group $G$ has finite rank then $E(G)$ is a finite dimensional rational algebra and so has minimum condition on left ideals. This is the case treated in [4]. However, it follows easily from a theorem of L. Fuchs [3] that there exist torsion free groups $G$ of infinite rank such that $E(G)$ is a finite dimensional rational algebra and Theorem 4.1 applies to these groups as well.

TheOREM 4.2. For any $\lambda \in E(G)$ there exists a decomposition $G \doteq H_{1} \oplus H_{2}$ such that $\lambda$ induces a quasi-automorphism on $H_{1}$ and $\lambda$ induces a nilpotent quasi-endomorphism on $\mathrm{H}_{2}$.

Proof. The mapping $\lambda^{*}: x \rightarrow x \lambda$ is an $E(G)$-endomorphism of the left $E(G)$-module $E(G)$ so that Fitting's Lemma for operator groups yields a module decomposition $E(G)=L_{1} \oplus L_{2}$ such that $\lambda^{*}$ is an automorphism on $L_{1}$ and is nilpotent on $L_{2}$. Let $L_{i}=E(G) e_{i}$ for orthogonal idempotents $e_{i}(i=1,2)$. By Lemma 2.5 we have $G \doteq H_{1} \oplus H_{2}$ with $H_{i} \doteq e_{i}(G)$.

Now, $\lambda e_{i} \in E e_{i}=L_{i}$ and $e_{i} \lambda \in L_{i} \lambda \subseteq L_{i}(i=1,2)$. Comparing the equations $\lambda=\lambda \cdot 1=\lambda e_{1}+\lambda e_{2}$ and $\lambda=1 \cdot \lambda=e_{1} \lambda+e_{2} \lambda$ we have $e_{i} \lambda$ $=\lambda e_{i}(i=1,2)$. Thus, $e_{i} \lambda=e_{i} \lambda e_{i} \in e_{i} E(G) e_{i}(i=1,2)$. The theorem follows easily now from Lemma 3.2, Theorem 3.3 and the wellknown fact that the elements of $\operatorname{Hom}_{E(G)}(E(G) e, E(G) e)$ are just the right multiplications by elements of $e E(G) e$.

CoRollary 4.3. If $G$ is strongly indecomposable then any quasiendomorphism of $G$ is either nilpotent or is a quasi-automorphism. 
CoROllary 4.4. If $G$ is strongly indecomposable then the sum of two nilpotent quasi-endomorphisms is again nilpotent.

Proof. As in Jacobson [5, p. 156].

One can use the corollaries above to show that the radical of $E(G)$ with $G$ strongly indecomposable coincides with the set of nilpotent elements. We remark finally that the results above can be extended to torsion free modules over an integral domain. We note, however, that the theorem of Fuchs referred to after the proof of Theorem 4.1 uses the Unique Factorization Theorem for the integers and, to the author's knowledge, would not be available should such an extension be made.

\section{BiBLIOGRAPHY}

1. R. A. Beaumont and R. S. Pierce, Torsion free rings, Illinois J. Math. 5 (1961), 61-98.

2. L. Fuchs, Abelian groups, Hungarian Academy of Sciences, Budapest, 1958.

3. - Indecomposable Abelian groups of arbitrary power, Acta Math. Acad. Sci. Hungar. 10 (1959), 453-457.

4. B. J6nsson, On direct decompositions of torsion free Abelian groups, Math. Scand. 7 (1959), 361-371.

5. N. Jacobson, Lectures in abstract algebra, Basic concepts, Vol. 1, Van Nostrand, New York, 1951.

6. - Structure of rings, Amer. Math. Soc. Colloq. Publ. Vol. 37, Amer. Math. Soc., Providence, R. I., 1956.

7. R. S. Pierce, Subrings of simple algebras, Michigan Math. J. 7 (1960), 241-243.

UNIVERSITY OF WASHINGTON AND SYRACUSE UNIVERSITY 